\title{
PROCESSOS HIDROLÓGICOS SUBSUPERFICIAIS INFLUENCIADOS POR CORTES DE ESTRADAS RURAIS NA BACIA HIDROGRÁFICA DO RIO GUABIROBA, GUARAPUAVA, PARANÁ, BRASIL
}

\author{
Márcia Cristina da Cunha \\ Universidade Federal de Jataí-UFJ \\ Unidade Acadêmica de Estudos Geográficos, Jataí, GO, Brasil \\ marcia1cunha@ufg.br \\ Edivaldo Lopes Thomaz \\ Universidade Estadual do Centro Oeste-UNICENTRO \\ Departamento de Geografia, Guarapuava, PR, Brasil \\ edilopes.thomaz@gmail.com
}

Fabiano Antonio de Oliveira Universidade Federal do Paraná-UFPR Departamento de Geografia, Curitiba, PR, Brasil foliveira@ufpr.br

\begin{abstract}
RESUMO
Embora as estradas rurais permitam o desenvolvimento das comunidades rurais, elas alteram a resposta hidrológica natural da bacia hidrográfica. Este estudo foi realizado na bacia do Rio Guabiroba, Guarapuava-PR, Brasil, com o objetivo de verificar como os processos hidrológicos subsuperficiais são influenciados por cortes de estrada rural. Para tanto, foram realizados levantamentos de campo para identificação dos tipos de interceptação do fluxo em subsuperfície. Foi realizado também o monitoramento da chuva $(\mathrm{mm})$, nível do lençol freático $(\mathrm{cm})$ e vazão $(\mathrm{l} / \mathrm{s})$ nos locais de interceptação. Como resultado, três tipos principais foram identificados: fluxo de retorno, fluxo pipe e fluxo insaturado. O fluxo do tipo pipe respondeu por mais de $60 \%$ do volume de vazão interceptada, porém, a altura da profundidade do nível do lençol freático e o tempo de permanência foi mais elevado no fluxo de retorno. Já o fluxo insaturado contribuiu com $20 \%$ do volume de vazão interceptada. Conclui-se, portanto, que os tipos de interceptação subsuperficial e os mecanismos diferenciados destes podem alterar a dinâmica hidrológica natural da bacia, provocando um tempo de resposta menor que por meio da infiltração e deslocamento no solo. Assim, estudos em estradas rurais é fundamental para o desenvolvimento das comunidades rurais do ponto de vista geográfico.
\end{abstract}

Palavras-chave: Bacia hidrográfica rural. Resposta hidrológica. Vertente. Interceptação.

\section{SUBSUPERFICIAL HYDROLOGICAL PROCESSES INFLUENCED BY CUTS OF RURAL ROADS IN THE GUABIROBA RIVER HYDROGRAPHIC BASIN, GUARAPUAVA-PARANÁ, BRASIL}

\begin{abstract}
Although rural roads allow the development of rural communities, they alter the natural hydrological response of the river basin. This study was carried out in the Guabiroba River Basin, Guarapuava-PR, Brazil, in order to verify how subsurface hydrological processes are influenced by rural road cuts. For that, field surveys were carried out to identify the types of interception of the flow in subsurface. Rainfall $(\mathrm{mm})$, groundwater level $(\mathrm{cm})$ and flow $(\mathrm{l} / \mathrm{s})$ were also monitored at the interception sites. As a result, three main types were identified: return flow, pipe flow and saturated troughflow. The pipe-type flow responded by more than $60 \%$ of the intercepted flow volume, however, the height of the water table level and the dwell time was higher in the return flow. The saturated troughflow contributed $20 \%$ of the volume of intercepted flow. It is concluded, therefore, that the types of subsurface interception and the differentiated mechanisms of these can alter the natural hydrological dynamics of the basin, provoking a shorter response time than through infiltration and displacement in the soil. Thus, studies on rural roads are fundamental for the development of rural communities from a geographical point of view.
\end{abstract}

Keywords: Rural catchment. Hydrologic response. Slope. Interception. 


\section{INTRODUÇÃO}

As estradas rurais são fundamentais para o desenvolvimento da sociedade (FAlZ et al., 2012), pois garantem a melhoria da qualidade de vida da população e, muitas vezes, essa é a única forma de acesso que a população tem aos serviços básicos disponibilizados em área urbanas, como saúde, educação, lazer, trabalho e outros. As estradas têm efeitos positivos e negativos. De um lado, são importantes para o desenvolvimento socioeconômico, especialmente nos países em desenvolvimento (FAIZ et al., 2012; SIDLE e ZIEGLER, 2012). Por outro lado, podem causar vários impactos sobre o ecossistema, tais como interceptação do fluxo subsuperficial.

A circulação da água é regulada e transferida nas vertentes basicamente por dois caminhos: fluxo em superfície e fluxo em subsuperfície. O fluxo em superfície, segundo Horton (1933), é aquele em que a água é rapidamente convertida em escoamento superficial até chegar aos cursos d'água naturais. Já a outra parte, infiltra no solo e, assim, de forma gradual, escoa através da matriz do solo, por meio dos pipes, escoamento insaturado e escoamento de retorno até o canal fluvial, ou perde-se por evapotranspiração para a atmosfera (ATKINSON, 1978; SIDLE e ZIEGLER, 2012; WEMPLE, 2013).

Nesse sentido, a crença de que o escoamento interno se movimenta lentamente para produzir volumes de água e somente o escoamento superficial seria o principal componente do hidrograma da bacia, nem sempre é verdadeira. Existe também o amplo apoio da comunidade hidrológica sobre a verticalidade da infiltração, desde a superfície até o nível freático (HARTSOG et al., 1997; FURNISS; LOVE; FLANAGAN, 1997; LUCE e WEMPLE, 2001; KOCHER; GERSTEIN; HARRIS, 2002; SIDLE e ZIEGLER, 2012; WEMPLE, 2013; THOMAZ; VESTENA; RAMOS-SCHARRÓN, 2014).

Por sua vez, o fluxo subsuperficial pode ser facilmente interceptado por cortes de estradas rurais, sendo um problema comum em muitos países (MENDIONDO, 1995; HARTSOG et al., 1997; LUCE e WEMPLE, 2001; SUNITHA et al., 2012). Por meio de vários mecanismos, as estradas interceptam a água do fluxo em subsuperfície em níveis significativamente potenciais para ampliar a rede de canal natural.

Em contraste com as formas fluviais naturais, o traçado das estradas rurais depende também dos fatores antropogênicos (WALDYKOWSKI e KRZEMIEŃ, 2013). O fluxo de água nas estradas nem sempre correspondem aos processos naturais que ocorrem nos leitos dos rios, tornando-se, assim, uma rede de drenagem artificial que complementa o sistema de drenagem natural da bacia hidrográfica (IMENSON, 1985; THOMAZ, VESTENA; RAMOS-SCHARRÓN, 2014; CUNHA e THOMAZ, 2017).

Assim, este tema cada vez mais vem ganhando destaque no meio científico. Já durante os anos de 1960 e 1970, da complexidade da geração do fluxo subsuperficial e o impacto que este gera começaram a aparecer em alguns trabalhos como, por exemplo, (WHIPKEY, 1965; KIRKBY e CHORLEY, 1967; BETSON e MARIUS, 1969; DUNNE e BLACK, 1970; WELC, 1972). E nas últimas décadas houve um interesse crescente por estudos relacionados à interceptação do fluxo subsuperficial por estradas rurais (JONES, 1981; HARTSOG et al., 1997; LUCE e WEMPLE, 2001; ANDREWS, et al., 2008; NEGISHI, et al., 2008; CHAPPELL, 2010; SIDLE e ZIEGLER, 2012; SIDLE; GHESTEM; STOKES, 2014; THOMAZ; VESTENA; RAMOS-SCHARRÓN, 2014; WEMPLE et al., 2017, CUNHA e THOMAZ., 2017).

A metodologia utilizada por esses autores é bastante variada: modelos de infiltração no solo, monitoramento de escoamento da chuva, modelagem de fluxo em pipe, monitoramento de efeitos hidrológicos e geomórficos em bacia rural, caracterização de processos em estradas, mapeamento de usos da terra (incluindo estradas), levantamento de sistema de drenagem entre outras. Contudo, ainda existe uma lacuna de estudos e procedimentos metodológicos que expliquem como os processos hidrológicos subsuperficiais podem ser influenciados por cortes em estradas rurais.

Assim, este estudo foi conduzido na bacia hidrográfica do Rio Guabiroba-PR, Brasil. A área de estudo é de extrema importância para o desenvolvimento da comunidade local, e além disso apresenta características geomorfológicas distintas, na qual permite estudos aprofundados sobre erosão de solos, estradas rurais, uso e ocupação da terra, sistema de drenagem, etc. A referida bacia é uma área experimental de estudos a mais de quinze anos da Universidade Estadual do Centro OesteUNICENTRO, com foco nas seguintes pesquisas: balanço hídrico (VESTENA, et al., 2012); controle de erosão em estradas (CUNHA, et al., 2013); concentração de sedimentos (THOMAZ; VESTENA;RAMOS-SCHARRÓN, 2014), qualidade ambiental (NEGRÃO e CUNHA, 2019) entre outros. 
O interesse crescente no fluxo subsuperficial e a erosão provocada por esse processo resultou em extensas revisões de pesquisas sobre o significado hidrológico e geomorfológico de fluxos em subsuperfície. Bryan e Jones (1997), Ziegler et al., (2001), Sidle e Ziegler (2012), ressaltam que um estudo global sobre como os processos geomorfológicos atuam e como os fluxos subsuperficiais são eficientes na transferência de água por meio da interceptação causada por estradas rurais, sedimento e solutos para as partes mais baixas das encostas e os canais fluviais é fundamental na prevenção dos impactos gerados por esse processo.

Constata-se, portanto, que as estradas alteram os processos geomorfológicos e hidrológicos e, por extensão, afetam o movimento de água e sedimento na bacia hidrográfica. Assim, os estudos em relação ao fluxo em subsuperfície vem tendo destaque nas pesquisas científicas. Contudo, ainda existe uma carência em explicar como os processos hidrológicos subsuperficiais podem ser alterados por cortes de estradas rurais. Portanto, o objetivo desde estudo foi verificar como os processos hidrológicos subsuperficiais são influenciados por cortes de estrada rural na bacia do Rio Guabiroba, Guarapuava, Paraná, Brasil.

\section{MATERIAIS E MÉTODOS}

\section{Área de estudo}

A bacia hidrográfica do Rio Guabiroba (Figura 1) possui uma área de $24 \mathrm{~km}^{2}$ (2.400 ha) sendo de $4^{\mathrm{a}}$ ordem de escoamento em escala de 1:10.000. A litologia predominante é constituída por basaltos da Formação São Bento (MINEROPAR, 2001). Quatro tipos de solo estão presentes na cobertura superficial: Latossolos, Cambissolos, Neossolos e Gleissolos (IUSS WORKING GROUP WRB, 2006). Além disso, ocorrem afloramentos de rochas em algumas seções das vertentes. Aproximadamente $75 \%$ da área apresenta inclinação entre $12 \%$ e mais de $30 \%$.

Figura 1 - Localização da bacia do Rio Guabiroba em relação ao município de Guarapuava.
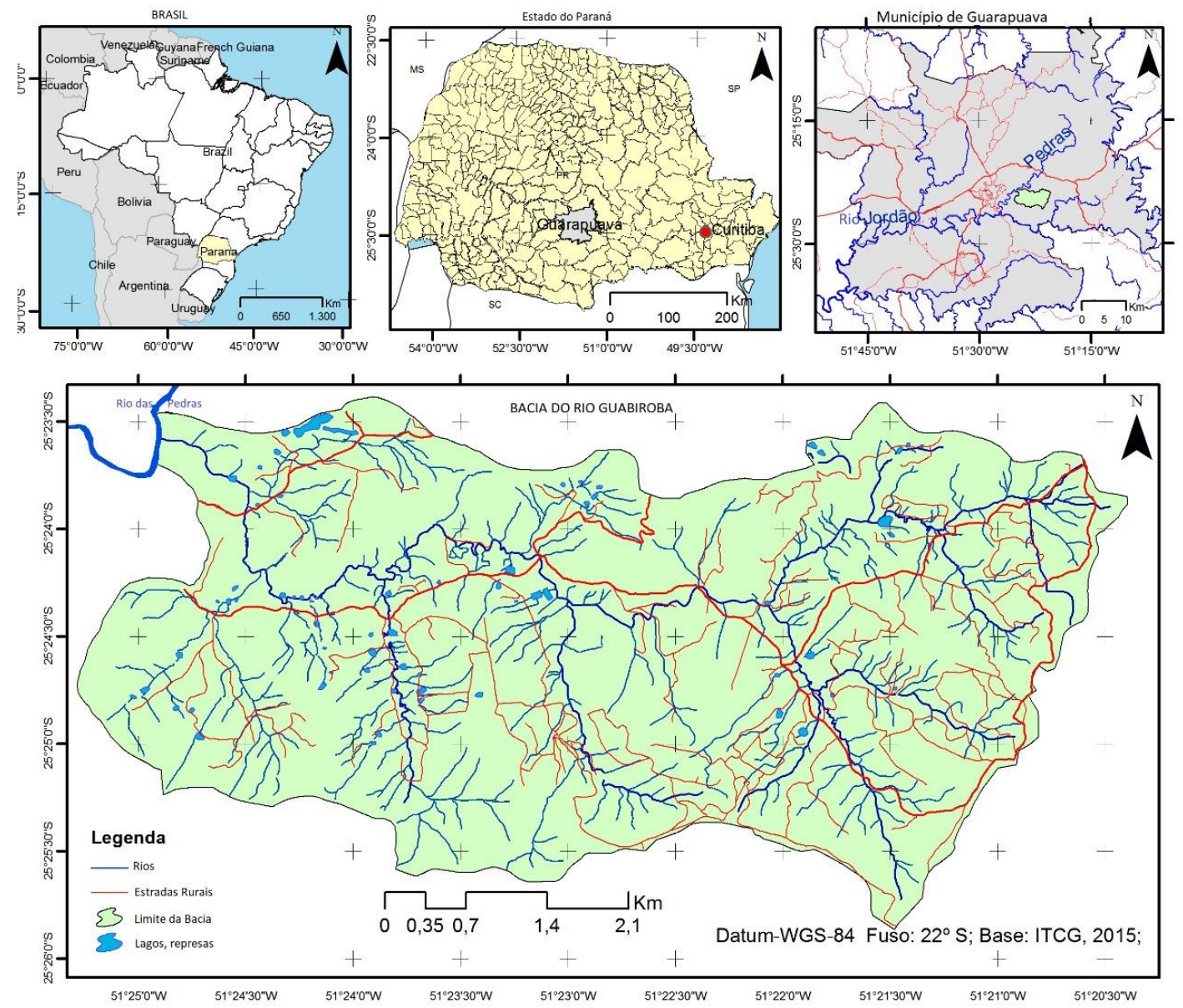

Fonte - França Júnior e Thomaz (2018). 
A área de estudo se enquadra na classificação climática de Köppen (1948) como mesotérmico úmido subtropical (Cfa). A temperatura média anual é de $17-18^{\circ} \mathrm{C}$. A precipitação é de $1.800-2.000 \mathrm{~mm}$, e a evapotranspiração anual é de 900-1.000 mm (CAVIGLIONE et al., 2000). O uso do solo não é diversificado, consistindo principalmente de floresta secundária $(53,9 \%)$, pastagem $(19,9 \%)$ e reflorestamento $(11,3 \%)$.

\section{MONITORAMENTO DOS LOCAIS DE INTERCEPTAÇÃO DO FLUXO SUBSUPERFICIAL}

O período total para identificação dos locais de interceptação do fluxo subsuperficial causado por cortes de estradas rurais foi 6 meses (dezembro de 2013 a maio de 2014). Nessa etapa percorreu-se todas as estradas da área de estudo, com um total de $130 \mathrm{~km}$ de estradas rurais. Após o período de identificação dos locais de interceptação, estes receberam classificação própria para diferenciação dos tipos de interceptação do fluxo em subsurperfície: fluxo de retorno, fluxo pipe e fluxo insaturado (Figura 2). A nomenclatura se baseou nas características que cada tipo de interceptação apresentou. No total foram monitorados oito locais individuais dentre os dos três tipos de interceptação observados.

Figura 2 - Tipos de interceptação do fluxo subsuperficial por estrada rural.

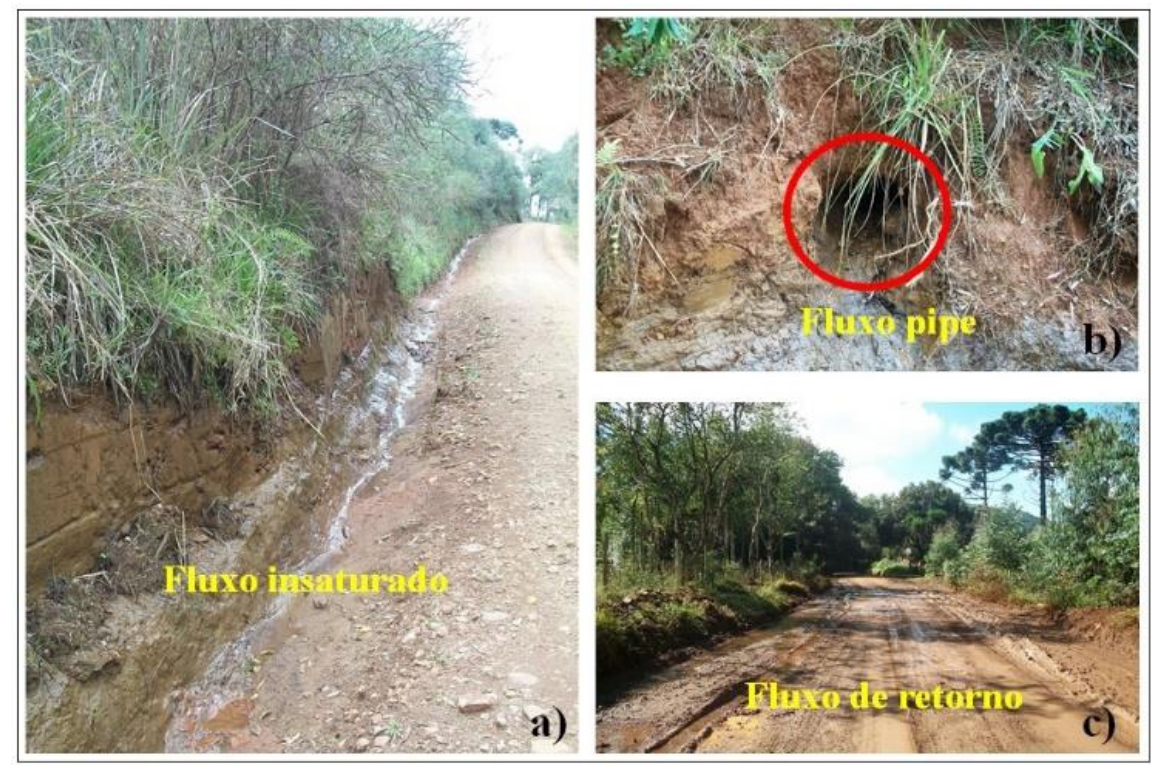

Em (a) interceptação do fluxo subsuperficial insaturado causado por estrada rural; (b) interceptação do fluxo subsuperficial pipe causado por estrada rural; (c) interceptação do fluxo subsuperficial de retorno causado por estrada rural.

Organizado pelos autores (2020).

- Fluxo insaturado: ocorre quando a micro-topografia não provoca um único canal. É a drenagem disseminada paralela à vertente por meio dos microporos existentes no solo que responde, geralmente, às mudanças nos gradientes hidráulicos. Nessa forma de fluxo subsuperficial não há uma hierarquia do fluxo. Prevalece em alta vertente, em relevo montanhoso com forma do terreno divergente-côncavo, que associado à presença de fragmentos de rocha resulta na dissipação da água no local.

- Fluxo pipe: são formados pela ação erosiva dos fluxos subsuperficiais que arrastam o material fino da matriz do solo. Esse processo envolve a progressiva expansão dos macroporos, principalmente devido à tensão de cisalhamento exercida pela água corrente. Esses poros podem ser formados por raízes de plantas, atividades da fauna, fissuras, rachaduras ou condutos naturais dentro do solo. $\mathrm{O}$ setor predominante desse tipo de interceptação é em média vertente com forma do terreno divergente-retilíneo em terreno suave ondulado, apresentando a dispersão do fluxo subsuperficial. 
- Fluxo de retorno: chamado de fluxo de retorno em subsuperfície (ou refluxo), ocorre depois que a água se infiltra no solo e, em seguida, rompe a superfície, emerge através do solo, e exfiltrando nas áreas de menor declive ou mais perto do canal fluvial, sendo mantidas pelas chuvas incidentes e pelo fluxo subsuperficial das áreas a montante. Ocorre geralmente em fundo de vale próximo ao canal fluvial ou em depressões úmidas. A forma do terreno é convergente-côncava, com relevo suave ondulado, facilitando assim o acúmulo e a permanência de água nos locais de interceptação.

\section{MONITORAMENTO DA CHUVA, VAZÃO E O NÍVEL DO LENÇOL FREÁTICO}

O monitoramento da chuva e do fluxo de interceptação (vazão) se deu no período de setembro de 2014 a agosto de 2015, no total de um ano. O monitoramento do nível do lençol freático ocorreu entre abril de 2015 a agosto do mesmo ano, totalizando cinco meses de monitoramento. Ressalta-se que os períodos de monitoramento da chuva, vazão e nível do lençol freático ocorreram em períodos diferentes em virtude da disponibilidade dos equipamentos e principalmente pela importância de 0 monitoramento compreender um período seco e chuvoso.

Para o monitoramento da chuva foram instalados três pluviógrafos do modelo Data Logging Rain Gauge RG3-M (figura 3a) distribuídos ao longo da bacia com o objetivo de coletar a chuva de forma distribuída na área estudada. No período monitorado foram registrados 79 eventos de chuva.

Para verificar o volume do fluxo subsuperficial interceptado (vazão) foram considerados o pico máximo atingido pela lâmina d'água em eventos de chuva em um período de 24 horas. Para isso foram instaladas Calhas Parshall (figura 3b) com gargantas de 9", com vazão mínima de $10 \mathrm{~m}^{3} / \mathrm{h}$ e vazão máxima de $925 \mathrm{~m}^{3} / \mathrm{h}$, calha com garganta de 2", com vazão mínima de $1 \mathrm{~m}^{3} / \mathrm{h}$ e vazão máxima de $60 \mathrm{~m}^{3} / \mathrm{h}$, e garganta de 6 ", com vazão mínima de $5 \mathrm{~m}^{3} / \mathrm{h}$ e vazão máxima de $400 \mathrm{~m}^{3} / \mathrm{h}$ instalada. A localização da instalação de cada calha se deu em função da observação do volume do fluxo nos locais monitorados. Para melhor interpretação dos dados os volumes de vazão foram transformados em I/s. No período monitorado foram registrados 60 eventos de vazão máxima.

Para monitoramento da profundidade do nível do lençol foram instalados piezômetros (figura 3c). No total foram registrados 19 eventos. Após a preparação dos piezômetros foram realizados furos de sondagem até alcançar a profundidade desejada (aproximadamente $1 \mathrm{~m}$ com diâmetro de $45 \mathrm{~mm}$ ), o que possibilitou a instalação dos tubos. Atingida a profundidade, colocamos na parte inferior dos furos uma pequena camada de areia média lavada, apoiando sobre essa camada o tubo já montado. A leitura do nível de água foi realizada por meio de uma régua acoplada a um suporte de madeira com $8 \mathrm{~mm}$ de diâmetro. A leitura da profundidade do nível do lençol freático a partir da superfície do solo foi realizada em média uma hora após o término da precipitação.

Figura 3 - Equipamentos instalados para monitoramento da chuva, nível do lençol freático e vazão.

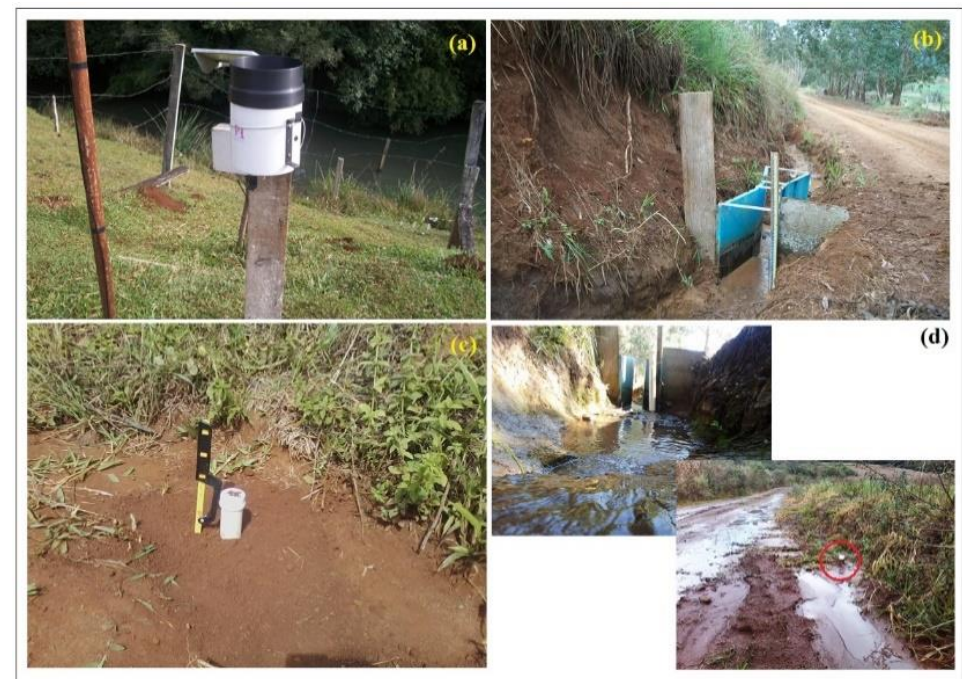

Em (a) pluviógrafo, em (b) calha parshall, em (c) piezômetro e em (d) calha registrando fluxo e piezômetro instalado em local de fluxo de retorno. Fonte - foto dos autores (2014-2015).

Organizado pelos autores (2020). 
Os dados analisados foram chuva $(\mathrm{mm})$, profundidade do nível do lençol freático (cm) e vazão máxima (I/S). A técnica de análise utilizada para avaliação dos dados foi a estatística descritiva (média e desvio padrão), baseando-se nos resultados obtidos da análise da amostra de oito locais de cada tipo de interceptação do fluxo em subsuperfície.

\section{RESULTADOS}

\section{MONITORAMENTO DOS LOCAIS DE INTERCEPTAÇÃO CAUSADO POR CORTES DE ESTRADAS RURAIS}

A interceptação do fluxo subsuperficial contribui para a conexão vertente e rio. Ocorre neste caso, a ligação entre as áreas separadas de alta e baixa vertente, em que o processo se inicia com a interceptação do fluxo insaturado, se intensifica no fluxo pipe e fecha o ciclo no fluxo de retorno em fundo de vale (Figura 4).

Figura 4 - Esquema dos tipos de interceptação do fluxo subsuperficial e da conexão vertente e rio. As flechas indicam a direção do fluxo.

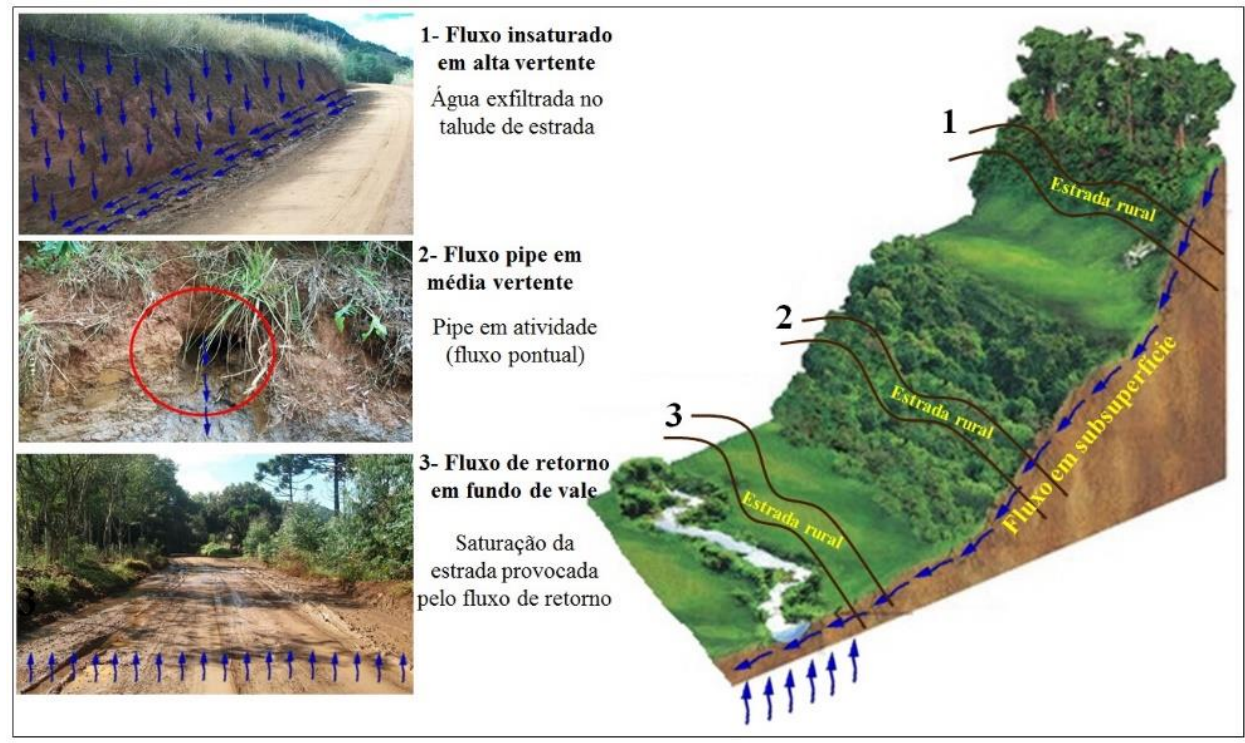

Organizado pelos autores (2020).

Uma das peculiaridades do sistema de estradas é que seu leito geralmente acompanha o fundo de vale (estradas principais), considerando que é mais viável a sua construção, pois sendo implantadas em tais condições utiliza-se a melhor localização e facilidades do terreno. Nesses locais a frequência de interceptação do fluxo de retorno é maior, ao passo que o fluxo pipe e insaturado se distribuem nas estradas secundárias e caminhos internos, que são construídas em todos os setores da bacia.

As estradas foram abertas inicialmente sem técnicas de implantação apropriada, por terem sido orientadas pela estrutura fundiária e pelas facilidades do terreno, originando, assim, pequenas trilhas e caminhos de acesso. Como consequência, ocorrem estradas encaixadas na encosta, taludes expostos, pistas sem controle de drenagem superficial e subsuperficial, ausência de vegetação em áreas de entorno, cruzamentos de estradas e rios, potencializando a degradação tanto da estrada como do canal fluvial.

\section{PRECIPITAÇÃO}

Registrou-se no total 79 eventos de chuva. O mês que apresentou maior volume pluviométrico foi jun/2014 com um total médio de 458,2 $227,6 \mathrm{~mm}$, (média baseada em três pluviógrafos) seguido pelo mês de jul/2015 com um total médio de $312,5 \pm 16,9, \mathrm{~mm}$. Os dois meses com menor pluviosidade

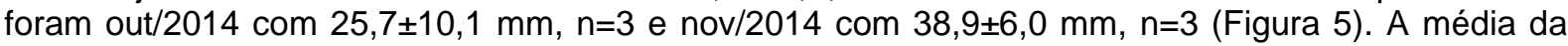
soma total de pluviosidade registrada nos três pluviógrafos foi de $2.340,4 \mathrm{~mm}$, no período monitorado. 
Figura 5- Total médio mensal de chuva registrada nos três pluviógrafos com os respectivos desvios padrão de cada mês monitorado.

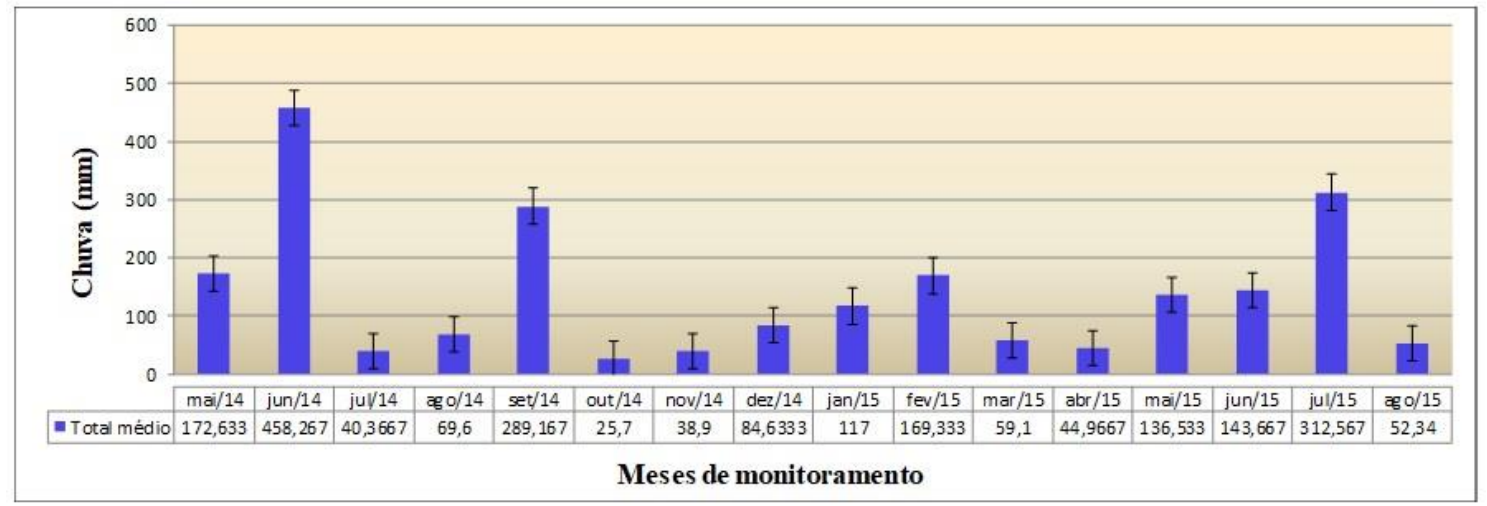

Organizado pelos autores (2020).

Os totais de chuva foram divididos em classes de $10 \mathrm{~mm}$ para verificar a frequência de eventos. Na Tabela 1, observa-se que a classe de chuva de 10-19,9 mm registrou 21 eventos (26,5\%), no entanto essa classe acumulou apenas $298,9 \mathrm{~mm}$ do total registrado. Por outro lado, a classe com evento igual ou superior a $50 \mathrm{~mm}$ teve um total de 13 eventos registrados (16,4\%), contudo acumulou 891,2 mm, respondendo, assim, por $38 \%$ da precipitação total do período.

Tabela 1 - Distribuição da precipitação por classe, total acumulado em mm e participação no total da precipitação.

\begin{tabular}{|c|c|c|c|c|c|}
\hline $\begin{array}{l}\text { Classe de } \\
\text { chuva }(\mathrm{mm})\end{array}$ & $\begin{array}{l}\text { Número de } \\
\text { eventos }\end{array}$ & $\begin{array}{c}\text { Intensidade } \\
\text { média } \\
(\mathrm{mm} / \mathrm{h})\end{array}$ & $\begin{array}{c}\text { Total de } \\
\text { precipitação } \\
\text { acumulada } \\
(\mathrm{mm})\end{array}$ & Frequência (\%) & $\begin{array}{c}\text { Precipitação } \\
\text { acumulada (\%) }\end{array}$ \\
\hline $1-9,9$ & 10 & 1,6 & 71,9 & 12,6 & 3,0 \\
\hline $10-19,9$ & 21 & 4,5 & 298,9 & 26,5 & 12,7 \\
\hline $20-29,9$ & 17 & 6,1 & 375,1 & 21,5 & 16,3 \\
\hline $30-39,9$ & 6 & 6,8 & 208,8 & 7,8 & 8,9 \\
\hline $40-49,9$ & 12 & 5,9 & 494,5 & 15,2 & 21,1 \\
\hline$\geq 50$ & 13 & 11,1 & 891,2 & 16,4 & 38,0 \\
\hline Total & 79 & 6,1 & $2.340,4$ & 100,0 & 100 \\
\hline
\end{tabular}

Fonte - dados coletados (2014/2015) e trabalhados pelos autores (2019/2020).

Portanto, os eventos com precipitações iguais ou superiores a $30 \mathrm{~mm}$ representam a menor quantidade registrada (31 eventos), entretanto em volume pluviométrico somado são os eventos que mais contribuem com precipitação, somando $1.594,5 \mathrm{~mm}(68,2 \%)$. Registrou-se também 48 eventos com precipitações inferiores a $30 \mathrm{~mm}$, que somam 745,9 mm $(31,8 \%)$ do total registrado.

A intensidade média dos eventos de chuva em relação à pluviosidade total foi de $6,1 \mathrm{~mm} / \mathrm{h}$, variando entre $1,6 \mathrm{~mm} / \mathrm{h}$ a $11,1 \mathrm{~mm} / \mathrm{h}$. O pico da precipitação, isto é, o período em que o volume de chuva foi maior, durou entre 50min e 7 h30min. Nesse intervalo, ocorreu em média $78 \%$ do volume total chuva dos eventos.

\section{NÍVEL DA PROFUNDIDADE DO LENÇOL FREÁTICO}

O nível da profundidade do lençol freático respondeu de forma positiva em relação à chuva nos locais monitorados ( $A=$ fluxo de retorno; $B=$ fluxo pipe e $C=$ fluxo insaturado) (Figura 6). As maiores elevações do nível do lençol freático corresponderam aos maiores volumes de chuva (87\%) da série monitorada, e os menores índices de chuva refletiram no nível do lençol no período de seca (13\%) da série (Figura 7). 
Figura 6 - Correlação entre precipitação e a profundidade do nível do lençol freático nos três pontos monitorados.
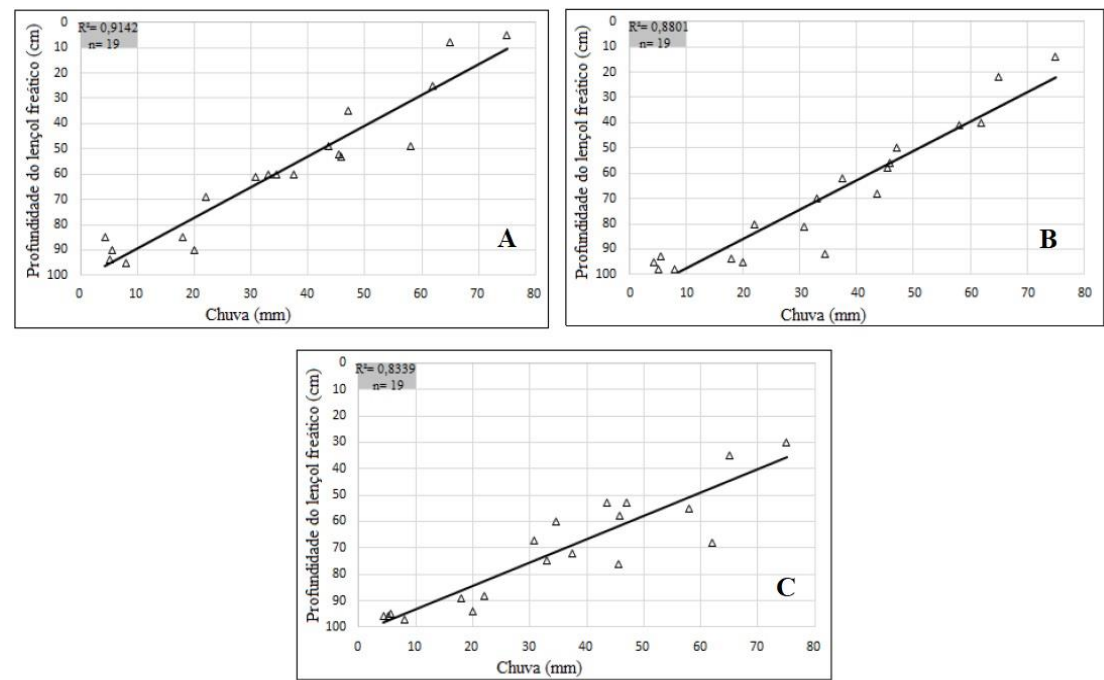

Organizado pelos autores (2020).

Figura 7 - Resposta média da profundidade do nível do lençol freático em relação a chuva nos locais monitorados na bacia do Rio Guabiroba.

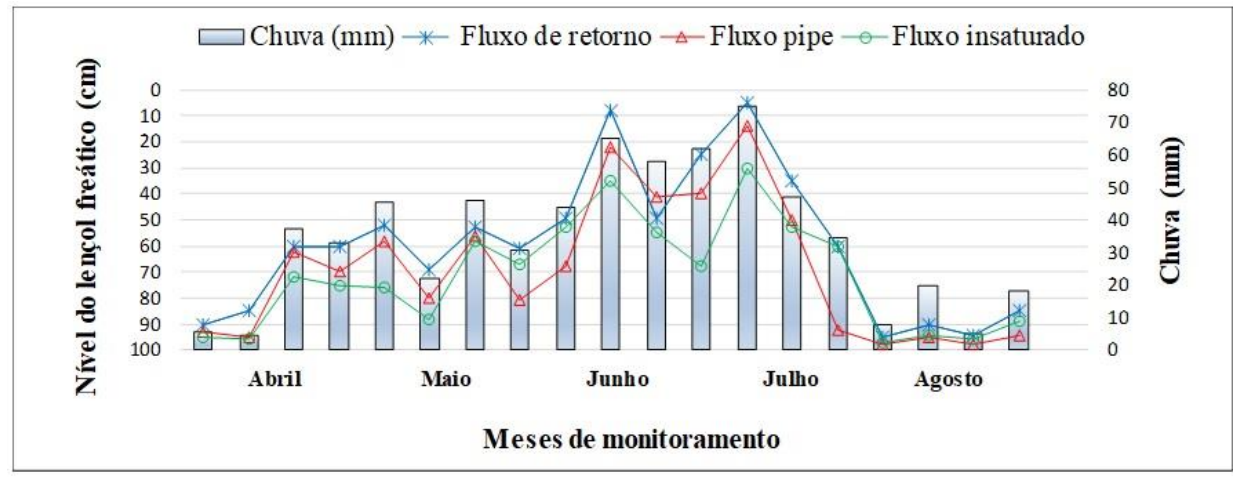

Organizado pelos autores (2020).

Houve variação entre o maior e o menor pico do nível do lençol freático (elevação e rebaixamento) nos locais de interceptação. Entretanto, os locais monitorados responderam de forma semelhante em relação à precipitação com elevação (período chuvoso) e rebaixamento (período seco) do nível do lençol freático (Tabela 2).

Tabela 2- Alteração da profundidade do nível do nível do lençol freático nos locais monitorados.

\begin{tabular}{lcccc}
\hline Nível lençol freático & $\begin{array}{c}\text { Dia/mês/ } \\
\mathbf{2 0 1 5}\end{array}$ & Fluxo de retorno & Fluxo pipe & $\begin{array}{c}\text { Fluxo } \\
\text { insaturado }\end{array}$ \\
\hline Média (cm) & - & $42,2 \pm 27$ & $31,2 \pm 25$ & $29,6 \pm 21$ \\
\hline Maior Elevação (cm) & $16 / j u l$ & 95 & 96 & 70 \\
$\begin{array}{l}\text { Maior Rebaixamento } \\
\text { (cm) }\end{array}$ & $24 / a g o$ & 04 & 02 & 04 \\
\hline
\end{tabular}

Fonte - dados coletados (2015) e trabalhados pelos autores (2019/2020).

No fluxo de retorno, a profundidade do nível do lençol freático respondeu de forma mais direta em relação à precipitação. Contudo, essa diferença quando comparada ao fluxo pipe é baixa. Nos três primeiros eventos de chuva que ocorreram em abril/2015 e nos quatro últimos eventos que ocorreram

$\begin{array}{lllll}\text { Caminhos de Geografia } \quad \text { Uberlândia-MG } & \text { v. 22, n. } 80 & \text { abr./2021 } & \text { p. 53-67 } & \text { Página } 60\end{array}$ 
em agosto/2015, foi observado o menor volume de chuva acumulada e, consequentemente, também a menor alteração do nível do lençol freático nos locais monitorados.

Já nos meses de maio, junho e julho/2015 registrou-se os maiores volumes de chuva e, assim, as maiores elevações do nível do lençol freático. Esses três meses juntos representaram mais de $87 \%$ do nível do lençol freático em toda a série analisada, ao passo que nos meses de abril e agosto responderam por pouco mais de $12 \%$ da série.

\section{VAZÃO}

Os índices de vazão representam de forma positiva nos períodos chuvoso e seco. Apesar disso, o fluxo pipe teve a maior contribuição com $61,4 \%$ (média $30,1 \pm 16,2 \mathrm{l} / \mathrm{s}$ ) de picos de vazão, ao mesmo tempo em que o fluxo insaturado teve contribuição de $20 \%$ (média $9,3 \pm 3,1 \mathrm{l} / \mathrm{s}$ ), e o fluxo de retorno 18,6\% (média de 7,1 $\pm 2,0 \mathrm{l} / \mathrm{s}$ ) (Figura 8).

Figura 8 - Média e valor mínimo e máximo de vazão nos locais monitorados.

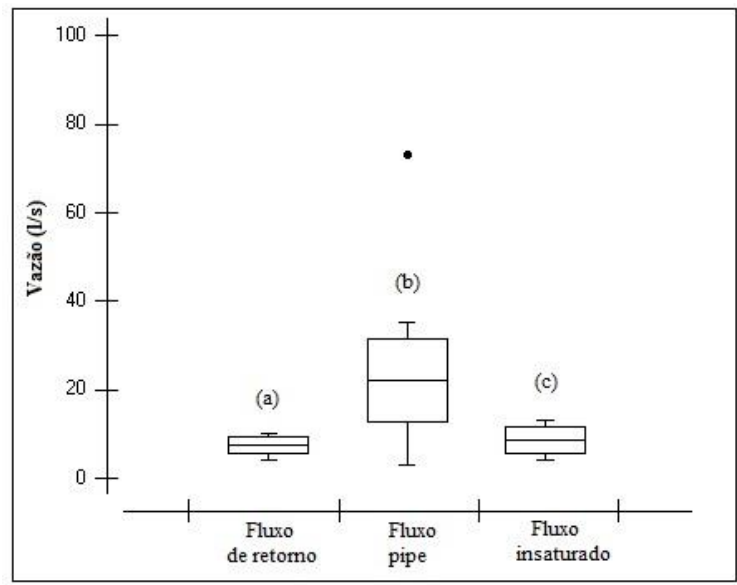

Organizado pelos autores (2020).

No fluxo pipe a variabilidade entre o valor mínimo e máximo e desvio padrão em relação à média dos picos de vazão foi superior comparada aos outros fluxos (amplitude 45,6 l/s), indicando mecanismos variados dentro do mesmo ambiente em resposta à precipitação com chuvas mais intensas. A resposta do fluxo pipe em relação a chuva foi mais forte comparado aos demais fluxos (Figura 9).

Figura 9 - Resposta acumulada da vazão na série monitorada em relação a chuva nos locais de interceptação do fluxo subsuperficial.

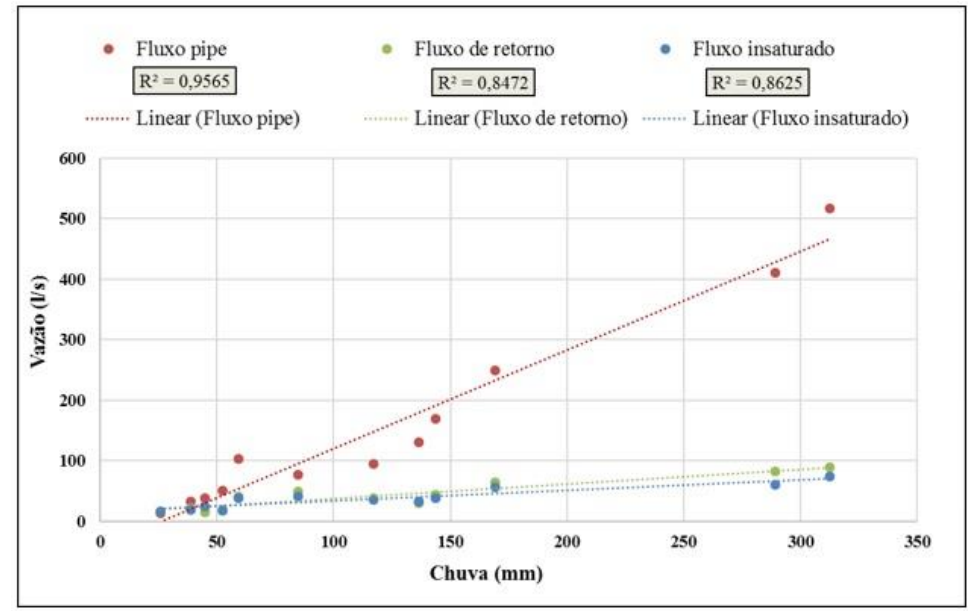

Organizado pelos autores (2020). 
No pipe $70 \%$ das vazões registradas foram iguais ou superiores a $20 \mathrm{l} / \mathrm{s}$, enquanto que no fluxo insaturado, $50 \%$ das vazões ficaram entre 13,4 e $10,6 \mathrm{l} / \mathrm{s}$ e os outros $50 \%$ corresponderam a vazões inferiores a $10 \mathrm{l} / \mathrm{s}$. Esse resultado é semelhante no fluxo de retorno em que $80 \%$ das vazões foram iguais ou inferiores a $10 \mathrm{l} / \mathrm{s}$ (Figura 10). O diâmetro entre um pipe e outro variou muito, tendo poucos centímetros (média de $4 \pm 4,2 \mathrm{~cm} \mathrm{n=5)}$ em alguns pontos e em outros podendo atingir mais de $50 \mathrm{~cm}$ (média de 18,8 $\pm 16,7 \mathrm{~cm} \mathrm{n}=3$ ).

Figura 10 - Permanência dos índices de vazão nos locais monitorados na bacia do Rio Guabiroba.

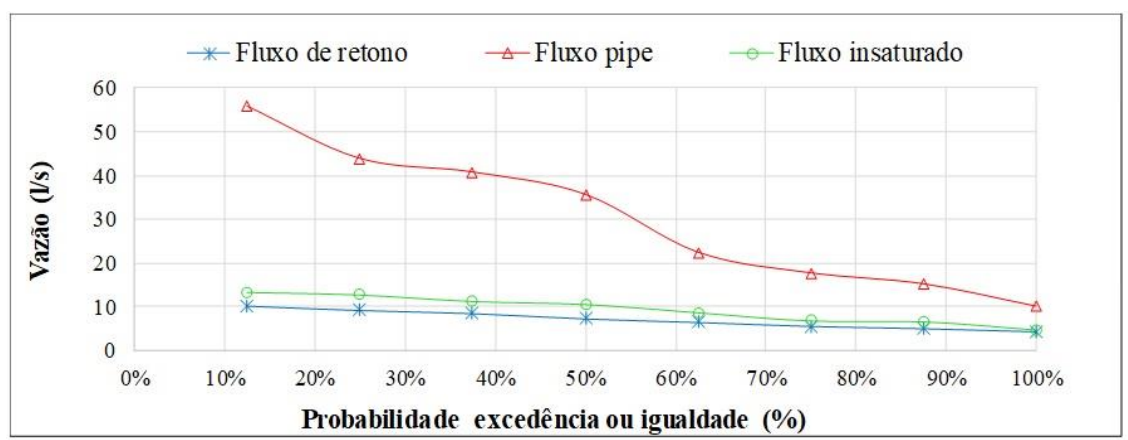

Obs. O tempo de permanência do fluxo interceptado refere-se a quantos dias o fluxo permanece em atividade após o cessar da precipitação, ou seja, o tempo de escoamento depois da interceptação causada pela estrada.

Organizado pelos autores (2020).

O tempo de permanência da vazão dos fluxos interceptados após o período de chuva oscilou entre os locais monitorados (Tabela 3 ). O fluxo de retorno permaneceu por mais tempo em atividade depois do cessar da chuva, seguido pelo fluxo insaturado. O fluxo pipe foi o que continuou por menor tempo em atividade após o período de chuva. Após a interceptação do fluxo subsuperficial nesses locais todos tornaram-se fluxo terrestre hortoniano, não ocorrendo a (re)infiltração no solo.

Tabela 3- Tempo de permanência de interceptação do fluxo subsuperficial

\begin{tabular}{cccc}
\hline TIPO DE FLUXO & Fluxo de retorno & Fluxo pipe & Fluxo insaturado \\
\hline Locais monitorado & $\begin{array}{c}\text { Tempo de fluxo em dias } \\
\text { de chuva consecutivos }\end{array}$ & $\begin{array}{c}\text { Tempo de fluxo em dias } \\
\text { de chuva consecutivos }\end{array}$ & $\begin{array}{c}\text { Tempo de fluxo em dias } \\
\text { de chuva consecutivos }\end{array}$ \\
\hline $\mathbf{1}$ & 15 & 10 & 8 \\
\hline $\mathbf{2}$ & 13 & 8 & 9 \\
\hline $\mathbf{3}$ & 12 & 6 & 5 \\
\hline $\mathbf{4}$ & 13 & 3 & 7 \\
\hline $\mathbf{5}$ & 9 & 7 & 8 \\
\hline $\mathbf{7}$ & 8 & 5 & 5 \\
\hline $\mathbf{8}$ & 10 & 4 & 6 \\
\hline Média & 7 & 4 & $\mathbf{6 , 8}$ \\
\hline
\end{tabular}

Fonte - dados coletados (2014-2015) e trabalhados pelos autores (2019/2020).

O fluxo pipe chama a atenção pelo fato de 30 eventos de vazão estarem entre 10 e $40 \mathrm{l} / \mathrm{s}$, representando, assim, $50 \%$ de vazão no período analisado. A chuva, para gerar esse volume de vazão, variou entre $10 \mathrm{~mm}$ e $50 \mathrm{~mm}$. Isso indica que nos locais monitorados não é necessário que a precipitação tenha muito volume, e um valor em torno de $40 \mathrm{~mm}$ com intensidade média de $5,8 \mathrm{~mm} / \mathrm{h}$ já é suficiente para gerar fluxo subsuperficial próximo ou superior a $20 \mathrm{l} / \mathrm{s}$.

$\begin{array}{lllll}\text { Caminhos de Geografia } \quad \text { Uberlândia-MG } & \text { v. 22, n. } 80 & \text { abr./2021 } & \text { p. } 53-67 & \text { Página } 62\end{array}$


Quanto ao fluxo de retorno e o fluxo insaturado, foi necessária uma precipitação mínima de $8 \mathrm{~mm}$ para gerar fluxo subsuperficial (vazão registrada), em média de 1,4 l/s, enquanto que chuvas em torno de $60 \mathrm{~mm}$ foram suficientes para gerar fluxo subsuperficial de 16,6 I/s. Já em relação ao fluxo pipe, a pluviosidade em torno de $12 \mathrm{~mm}$ foi suficiente para gerar vazões de $16,6 \mathrm{l} / \mathrm{s}$, ou seja, um volume de chuva cinco vezes inferior do fluxo de retorno e insaturado foi suficiente para gerar a mesma quantidade de vazão no fluxo pipe.

\section{DISCUSSÃO}

Os resultados do estudo fornecem várias implicações importantes para o gerenciamento de estradas rurais em bacias hidrográficas, onde as características dos locais de implantação das estradas e os processos de geração de fluxo subsuperficial são condizentes com a interceptação deste pela estrada (LUCE e WEMPLE, 2001; WEMPLE, 2013; THOMAZ; VESTENA; RAMOS-SCHARRÓN, 2014). Além de atuar como fonte do escoamento superficial, as estradas também interceptam o fluxo subsuperficial. Vale ressaltar que a resposta do fluxo subsuperficial interceptado foi altamente dependente dos efeitos pluviométricos e geomórficos da área estudada.

A infiltração e a percolação envolvem três processos interdependentes: entrada de água no topo do solo, armazenamento dentro do solo e transmissão de água através do solo. Qualquer alteração em um desses três processos modifica a resposta hidrológica de uma área, principalmente a alteração na transmissão da água no solo por meio da interceptação do fluxo subsuperficial causado por cortes das estradas (DUNNE, 1978; IMENSON, 1985; CUNHA e THOMAZ, 2017). Na área de estudo, esses processos foram alterados por meio da interceptação do fluxo subsuperficial causado pelas estradas rurais, sobretudo o fluxo pipe que responde por $61,4 \%$ do total da vazão interceptada.

Em um estudo realizado na bacia experimental Bukit Tarek na Malásia, Negishi et al., (2008) durante o monitoramento de um ano, constataram que a estrada interceptou um volume substancial de fluxo subsuperficial, com sua contribuição aumentando para mais de $90 \%$ do total escoamento. Consequentemente, para as 11 tempestades intensamente monitoradas, os sedimentos derivados do fluxo em subsuperfície representaram $28 \%$ do total sedimento exportado da seção de estrada.

Contudo, em um outro estudo realizado no norte da Tailândia na bacia hidrográfica de Pang Khum Village, Ziegler et al., (2001), não conseguiram encontrar evidência de que a interceptação do fluxo subsuperficial na estrada rural contribui substancialmente para o escoamento da área de estudo. Neste caso, existe um indício de que a interceptação do fluxo subsuperficial causado por estradas rurais, não se aplica em todas as bacias hidrográficas.

$\mathrm{Na}$ área de estudo, verificou-se que no fluxo pipe volumes de chuva em torno de $40 \mathrm{~mm}$ com intensidade média de $5,8 \mathrm{~mm} / \mathrm{h}$ foram suficientes para gerar fluxo subsuperficial próximo ou superior a $20 \mathrm{l} / \mathrm{s}$, ou seja, volumes de chuva inferior a $83,3 \%$ são suficientes para gerar a mesma quantidade de vazão máxima nos outros dois tipos de fluxo monitorados. A dinâmica diferenciada nesse tipo de interceptação pode ser um indicativo da resposta em relação à forma de interceptação e a localização da vertente, facilitando a dissipação da água no local monitorado (JONES e GRANT, 1996; THOMAS e MEGAHAN 1998).

Em um estudo feito no Brasil, os diâmetros de pipes encontrados nas sub-bacias do Arroio TurcatoRS, variaram entre 2 e $12 \mathrm{~cm}$ e o escoamento permaneceu vários dias após ocorrência de chuvas. Dessa forma, as vazões observadas na saída de uma rede de pipe, no período de março a agosto de 1996, variaram de $5,5 \mathrm{l} / \mathrm{min}$ a $12,4 \mathrm{l} / \mathrm{min}$ (MENDIONDO, 1995).

Quando a água percolada verticalmente encontra valores de condutividade hidráulica menores à medida que varia com a profundidade, ocorre então a formação de uma camada de saturação. Nesse caso o nível da profundidade do lençol freático foi mais elevado no fluxo de retorno com $45,5 \% \mathrm{~cm}$ de altura do total monitorado (MENDIONDO e TUCCI, 1997). Isso é resultado do processo da saturação do solo e da exfiltração do fluxo subsuperficial interceptado pela estrada no local. Como consequência, ocorre saturação prolongada do solo, permanecendo em atividade entre uma precipitação e outra no período chuvoso, com rebaixamento de poucos centímetros da profundidade do nível do lençol freático no local no período seco.

Portanto, quando comparado à resposta hidrológica dos locais de interceptação após o início da chuva, esta foi mais acelerada no fluxo pipe e no fluxo insaturado, e mais lenta no fluxo de retorno. 
Esses dois processos acelerados são comandados pela vertente, não dependendo necessariamente da ascensão do nível do lençol freático, em que a conexão vertente e rio é mais instantânea do que no fluxo de retorno, já que a água escoa em velocidade por meio dos macroporos.

O fluxo de retorno, porém, necessita que a água da vertente chegue até o fundo de vale e também que a água da subsuperfície da vertente reflita no aumento da vazão do rio para que tenha ascensão do lençol freático, aumentando assim o tempo de resposta do processo (JONES, 1981; CHAPPELL, 2010; SIDLE e ZIEGLER, 2012; SIDLE; GHESTEM; STOKES, 2014).

A variabilidade espaço-temporal do fluxo subsuperficial que é interceptado por estradas também depende da profundidade do corte da estrada em relação à zona de saturação do solo e fluxo subsuperficial (tipicamente o contato solo-leito de rocha). Exemplo disso é nas regiões montanhosas de Idaho, EUA, onde a saturação do solo é suficiente para gerar fluxo em subsuperfície lateral de estradas, em que 0 processo se intensifica principalmente durante 0 derretimento da neve (BURROUGHS; MARSDEN; HAUPT, 1972).

Portanto, os resultados atuais são consistentes com a noção de que a probabilidade de ocorrência, e resposta do fluxo interceptado, também depende de como as estradas são implantadas em relação a vertente (por exemplo, alta, média e baixa vertente). (TSUKAMOTO e OHTA, 1998; LUCE, 2002; MIRUS et al., 2007). Assim, estudos relacionados a interceptação do fluxo subsuperficial são extremamente importantes do ponto de vista geográfico uma vez que podem contribuir de forma direta com o desenvolvimento da sociedade, em especial das comunidades rurais.

\section{CONSIDERAÇÕES FINAIS}

O leito das estradas rurais, quando aprofundados, interceptam o fluxo subsuperficial, o que faz essas vias importantes componentes na circulação de água. Tornam-se uma rede hidrográfica acessória da rede de drenagem natural em eventos de chuva, aumentando significativamente a densidade de drenagem da bacia em $60 \%$. Exerceram então, papel fundamental na conexão vertente e rio e na dissecação do solo, sendo eficientes na transmissão do fluxo subsuperficial que foi interceptado.

Identificou-se três tipos principais de interceptação de fluxo em subsuperfície. O fluxo insaturado e o fluxo de retorno apresentam semelhanças em relação ao volume de vazão interceptada. O fluxo pipe se destaca por responder por mais de $60 \%$ do volume de vazão interceptada, em que a resposta hidrológica é mais rápida facilitando a dissipação da água interceptada. Contudo, o tempo de atividade após a chuva é mais elevado no fluxo de retorno por ocorrer em lençol freático raso ou áreas saturadas próximo à rede de drenagem, em nível de base e pressão hidrostática.

Portando, os mecanismos diferenciados do funcionamento hidrológico de áreas interceptadas podem alterar a dinâmica natural da bacia hidrográfica, produzindo então resposta hidrológica menor que por meio da infiltração e transferência de fluxo interno no solo, conectando de forma acelerada vertente e rio. Sugere-se neste caso, que os estudos de processos hidrogeomorfológicos em estradas rurais sejam consideradas no planejamento dos recursos naturais.

Os estudos sobre processos hidrológicos subsuperficiais interceptados por cortes de estradas rurais, é fundamental para a compreensão conjunta dos elementos da paisagem presentes em bacias rurais, uma vez que auxiliam na diminuição dos impactos oriundos dessa dinâmica diferenciada de drenagem na bacia. Sendo assim, o estudo atingiu o objetivo proposto que foi verificar como os processos hidrológicos subsuperficiais são influenciados por cortes de estrada rural. Entretanto, colocam-se perspectivas futuras na linha de estudo. O artigo tratou da interceptação do fluxo em subsuperfície causado por estradas rurais, no entanto há necessidade de explicar como as estradas rurais evoluíram no decorrer dos anos, e se com isso houve um aumento gradativo na interceptação do fluxo? Com o aumento rápido da vazão somado ao fluxo interceptado, ocorre também o aumento do nível de turbidez no canal fluvial? A interceptação do fluxo subsuperficial causado pelas estradas, pode ser influenciado pelos diferentes usos da bacia?

\section{AGRADECIMENTOS}

A Coordenação de Aperfeiçoamento de Pessoal de Nível Superior (CAPES) por ter concedido bolsa. 


\section{REFERÊNCIAS}

ANDREWS, K. M.; GIBBONS, J. W.; JOCHIMSEN, D. M.; MITCHELL, J. (2008). Ecological effects of roads on amphibians and reptiles: A literature review. Herpetological Conservation, v. 3, p. 121-143, 2008.

ATKINSON, C. Techniques for Measuring Subsurface Flow in Hillslopes. In: KIRKBY, M. (ed.) Hillslope Hydrology, New York: John Wiley \& Sons, p.73-120, 1978.

BAESSO, D. P.; GONÇALVES, F. L. R. Estradas não pavimentadas: técnicas adequadas de manutenção. Florianópolis: DER, 2003. 204 p.

BETSON, R. P.; MARIUS, J. B. Source areas of storm runoff. Water Resour. v. 5, p. 574-582, 1969. https://doi.org/10.1029/WR005i003p00574

BONELL, M.; HENDRIKS, M.; IMESON, A.; HAZELHOFF, L. The Generation of Storm Runoff in a Forested Clayey Drainage Basin in Luxembourg. Journal of Hydrology, Amsterdam, v.71, p.53-77, 1984. https://doi.org/10.1016/0022-1694(84)90071-4

BRYAN, R. B.; JONES, J. A. A. The significance of soil piping processes: inventory and prospect. Soil Erosion Laboratory, University of Toromo, Scarborough, Ont. M1C IA4, Canadá. Geomorphology., v. 20, n.4, p. 209-218, 1997. https://doi.org/10.1016/S0169-555X(97)00024-X

BURROUGHS E. R.; MARSDEN M. A.; HAUPT H. F. Volume of snowmelt intercepted by logging roads. Journal of Irrigation and Drainage Division of the American Society of Civil Engineers $n$. 98: p. 1-12, 1972. https://doi.org/10.1061/JRCEA4.0000852

CAVIGLIONE, J. H.; KIIHL, L. R. B.; CAMAROTI, P. H.; OLIVEIRA, A. D. Cartas climáticas do Paraná. Londrina: IAPAR-Instituto Agronômico do Paraná, 2000.

CHAPPELL, N. A. Soil pipe distribution and hydrological functioning within the humid tropics: a synthesis. Lancaster Environment Centre, Lancaster University, Lancaster LA1 4YQ, UK. Hydrol. Process. v. 24, p. 1567-1581, 2010. https://doi.org/10.1002/hyp.7579

CROKE, J.; MOCKLER, S. Gully initiation and road-to-stream linkage in a forested catchment, southeastern Australia. Earth Surface Processes and Landforms, v. 26, n. 2, p. 205217, 2001. https://doi.org/10.1002/1096-9837(200102)26:2<205::AID-ESP168>3.0.CO;2-G

CUNHA, M. C.; THOMAZ, E. L. Fluxo subsuperficial interceptado por estrada rural: características e distribuição na paisagem. Bol. Goia. Geogr. (Online). Goiânia, v. 37, n. 3, p. 429-447, set./dez. 2017. https://doi.org/10.5216/bgg.v37i3.50763

DUNNE, T. Field Studies of Hillslope Flow Processes In: KIRKBY, M. (ed.) Hillslope Hydrology, New York: John Wiley \& Sons, p.227-293, 1978.

. BLACK, R. D. Partial area contributions to storm runoff in a small New https://doi.org/10.1029/WR006i005p01296

FAIZ, A. et al. Sustainable rural roads for livelihoods and livability. Procedia - Social and Behavioral Sciences., v. 53, n.1, p. 1-8, 2012. https://doi.org/10.1016/j.sbspro.2012.09.854

FRANÇA JUNIOR, P.; THOMAZ, E. L. Land-use changes and the increase in the number road-stream crossings in a rural basin south of Brazil. Revista Brasileira de Geomorfologia, v. 19, n. 4, 2018. https://doi.org/10.20502/rbg.v19i4.1380

FURNISS, M. J.; LOVE, M.; FLANAGAN, S. A. Diversion Potential at Road-Stream Crossings. United States Department of Agriculture Forest Service Technology e Development Program, 1997.

GONÇALVES, J. L. M. Conservação do solo. In. GONÇALVES, J. L. M. STAPE, J. L. (ed). Conservação e cultivo de solos para plantações florestais. Piracicaba: IPEF, 2002. cap. 2, p. 47129.

HARTSOG, W.; KAHKLEN, K.; MOLL, J.; SWANSTON, D. N. A Monitoring System for Measuring Effects of Roads on Groundwater: Equipment and Installation. United States Department of Agriculture Forest Service Technology e Development Program, 1997.

HORTON, R. E. The Rôle of infiltration in the hydrologic cycle, Eos Trans. AGU, 14 (1), 446-460, 1933. https://doi.org/10.1029/TR014i001p00446

$\begin{array}{lllll}\text { Caminhos de Geografia } \quad \text { Uberlândia-MG } & \text { v. 22, n. } 80 \quad \text { abr./2021 } & \text { p. 53-67 Página } 65\end{array}$


IMENSON, A. L. Geomorphological processes, soil structure and ecology. In: PITTY, A. Themes on Geomorphology. London: Croomhelm, 1985.

IUSS WORKING GROUP WRB. World reference base for soil resources-a framework for international classification, correlation and communication. World Soil Resources Reports 103. Food and Agriculture Organization of the United Nations, Rome, 2006.

JONES, A. A. The Nature of Soil Piping, a Review of Research. Geobooks, Norwich, p. 301, 1981.

GRANT, G. E. Peak flow responses to clear-cutting and roads in small and large basins, western Cascades, Oregon. Water Resources Research v. 32, p. 959974, 1996.

KIRKBY, M. J.; CHORLEY, R. Throughflow, overland flow and erosion. Bull. Int. Assoc. Sci. Hydrol. v. 12, n. 3, p. 5-21, 1967.

KNEALE, W. The Hydrology of a Sloping, Strutured Clay Soil at Wytham, NearOxford, England, Amsterdam, Journal of Hydrology, v. 85, p. 1-14, 1996. https://doi.org/10.1016/0022-1694(86)90073$\underline{9}$

KOCHER, S. D.; GERSTEIN, J. M.; HARRIS, R. R. Rural Roads. A Construction and Maintenance Guide for California Landowners, Berkeley, University of California, 2002.

KÖPPEN, W. Climatologia. México, Fundo de Cultura Econômica, 1948.

LUCE, C. H. Hydrological processes and pathways affected by forest roads: what do we still need to learn? Rocky Mountain Research Station, Boise Aquatic Sciences Laboratory, 316 E. Myrtle, Boise, ID 83702, USA Hydrol. Process. v. 16, 2901-2904, 2002.

WEMPLE, B. C. Introduction to special issue on hydrologic and geomorphic of forest roads. Earth Surf. Process. Landf., v. 2, n. 8, p. 111-113, 2001. https://doi.org/10.1002/10969837(200102)26:2<111::AID-ESP165>3.0.CO;2-2

MENDIONDO, E. D. Integração das Escalas Hidrológicas nas Sub-Bacias Embutidas do Rio Potiribú, RS. Dissertação (Mestrado Em Recursos Hídricos e Saneamento Ambiental), UFRGS. Porto Alegre: IPH, 1995.

TUCCI, C. E. M. Escalas hidrológicas II: diversidade de processos na bacia vertente. São Carlos, SP. Revista Brasileira de Recursos Hídricos, ABRH, v. 2, n. 1, p. 59-122, 1997. https://doi.org/10.1163/22224297-90000162

MINEROPAR - Serviço Geológico do Paraná. Atlas Geológico do Estado do Paraná. Curitiba: Serviço Geológico do Paraná, 2001.

MIRUS, B. B.; EBEL, B. A.; LOAGUE, K.; WEMPLE, B. C. Simulated effect of a forest road on nearsurface hydrologic response: redux. Earth Surface Processes and Landforms. n. 32: 126-142, 2007. https://doi.org/10.1002/esp.1387

NEGISHI, J. N.; SIDLE, R. C.; ZIEGLER, A. D.; NOGUCHI, S.; RAHIM, N. A. Contribution of intercepted subsurface flow to road runoff and sediment transport in a logging-disturbed tropical catchment. Earth Surf. Process. Landforms v. 33, 1174-1191, 2008. https://doi.org/10.1002/esp.1606

NEGRÃO, G. N.; CUNHA, M. C. Diversidade de macroinvertebrados bentônicos na avaliação do uso do solo e qualidade ambiental da bacia do Guabiroba, Guarapuava, PR, Brasil. Curitiba, PR. Revista Geografar., v. 14, n 01, p. 07-26, 2019. https://doi.org/10.5380/geografar.v14i1.53497

SIDLE, R. C.; GHESTEM, M.; STOKES, A. Epic landslide erosion from mountain roads in yunnan, China-challenges for sustainable development. Nat. Hazards Earth Syst. Sci, v. 14, p. 3093-3104, 2014. https://doi.org/10.5194/nhess-14-3093-2014

ZIEGLER, A. D. The dilemma of mountain roads. Nature Geoscience., v. 5, n.7, p. 437438, 2012. https://doi.org/10.1038/ngeo1512 
SUNITHA, V.; VEERARAGAVAN, A.; KARTHIK, K. S.; MATHEW, S. Clusterbased pavement deterioration models for low-volume rural roads. International Scholarly Research Network ISRN Civil Engineering Volume Article ID 565948, 2012. https://doi.org/10.5402/2012/565948

THOMAS, R. B.; MEGAHAN, W. F. Peak flow responses to clearcutting and roads in small and large basins, western Cascades, Oregon: a second opinion. Water Resources Research v. 34, p. 3393-3403, 1998. https://doi.org/10.1029/98WR02500

THOMAZ, E. L.; VESTENA, L. R.; RAMOS-SCHARRÓN, C. E. The effects of unpaved roads on suspended sediment concentration at varying spatial scales - a case study from Southern Brazil. WEJ (Hertford)., v. 28 n.4, p. 547-555, 2014. https://doi.org/10.1111/wej.12070

TSUKAMOTO, Y.; OHTA, T. Runoff process on a steep forested slope. Journal of Hydrology. n. 102: 165-178, 1998. https://doi.org/10.1016/0022-1694(88)90096-0

VESTENA, L.; OLIVEIRA, E. D. de.; CUNHA, M. C. C.; THOMAZ, E. L. Vazão ecológica e disponibilidade hídrica na bacia do Rio das Pedras, Guarapuava-PR. Revista Ambiente \& Água, v. 7 , p. 212-227, 2012. https://doi.org/10.4136/ambi-agua.840

WALDYKOWSKI, P.; KRZEMIEŃ, K. The role of road and footpath networks in shaping the relief of middle mountains on the example of the Gorce Mountains (Poland). Gebr. Borntraeger Verlagsbuchhandlung, Stuttgart, Germany. Zeitschrift für Geomorphologie v. 57, n. 4 p. 429-470, 2013. https://doi.org/10.1127/0372-8854/2013/0108

WELC, A. Transportation of suspended matter in the Rivers Ropa and Bystrzanka and magnitude of wash down during the flood in July 1970. Studia Geomorph. Carpatho, Balcan. v. 6, p. 206-209, 1972.

WEMPLE, B. C. Assessing the Effects of Unpaved Roads on Lake Champlain Water Quality. Lake Champlain Basin Program. Technical report n. 74, 2013. https://doi.org/10.1002/eco.1881

et al., Ecohydrological disturbances associated with roads: Current knowledge, research needs, and management concerns with reference to the tropics. Ecohydrology. 2017; e1881.

WHIPKEY, R. Z. Subsurface stormflow from forested slopes. Bull. Int. Assoc. Sci. Hydrol. v. 10, p. 74-85, 1965. https://doi.org/10.1080/02626666509493392

ZIEGLER, D. A.; GIAMBELLUCA, T. W.; SUTHERLAND, R. A;. VANA, T. T.; NULLET, M. A. Horton overland flow contribution to runoff on unpaved mountain roads: A case study in northern Thailand. Scientific Briefing Hydrol. Process. v. 15, 3203-3208, 2001. https://doi.org/10.1002/hyp.480

Recebido em: 08/05/2020

Aceito para publicação em: 22/10/2020 